\title{
Closed-loop phase equilibria of a symmetrical associating mixture of square-well molecules examined by Gibbs ensemble Monte Carlo simulation
}

\author{
Lowri A. Davies \\ Department of Chemistry, University of Sheffield, Sheffield S3 7HF, United Kingdom \\ George Jackson \\ Department of Chemical Engineering and Chemical Technology, Imperial College of Science, Technology and Medicine, \\ Prince Consort Road, London SW7 2BY, United Kingdom \\ Luis F. Rull \\ Departamento de Física Atómica Molecular y Nuclear, Universidad de Sevilla, Apartado 1065, Sevilla 41080, Spain
}

(Received 21 June 1999)

\begin{abstract}
A closed loop of liquid-liquid immiscibility for a simple model binary symmetrical mixture of square-well monomers with a single short-ranged interaction site has been recently observed using the Gibbs ensemble Monte Carlo technique [L. A. Davies, G. Jackson, and L. F. Rull, Phys. Rev. Lett. 82, 5285 (1999)]. This model system has unfavorable mean-field interactions between unlike components which leads to phase separation at intermediate temperatures; the addition of a directional bonding site leads to association and miscibilty of the system at low temperatures. In this work we present a detailed study of the effect of a variation in pressure and of the strength of the bonding interaction on the phase equilibria of such a model system by Gibbs ensemble simulation. The phase diagram is dominated by regions of liquid-liquid immiscibility which are bounded at high temperatures by an upper critical solution temperature and by a lower critical solution temperature (LCST) for specific values of the pressure and association strength. This closed-loop region is seen to increase in size as the pressure of the system is increased. For weak bonding interaction strengths the system does not possess a LCST and is seen to exhibit regions of two-phase vapor-liquid coexistence which are separated from the region of liquid-liquid immiscibility by a three-phase line. The phase equilibria of the same model system is also determined using the statistical associating fluid theory as adapted for potentials of variable range; the theory provides a good description of the closed-loop immiscibility and other features of the phase diagram.
\end{abstract}

PACS number(s): 64.60.-i, 61.20.Qg, 05.20.Jj

\section{INTRODUCTION}

The existence of closed-loop regions of liquid-liquid immiscibility in the phase diagrams of aqueous fluid mixtures such as aliphatic and aromatic alcohols [1], amines [2], and nonionic surfactants [3] can be readily understood in terms of the nature of the intermolecular interactions. The association of unlike species via directional interactions such as hydrogen bonds leads to the low-temperature miscibility of the system. These bonds break as the temperature of the system increases resulting in phase separation above the lower critical solution temperature (LCST) due to the residual weak unlike interactions. The increase in the kinetic energy, and hence the entropy of the molecules at higher temperatures ensures that the system is miscible above the upper critical solution temperature (UCST). A detailed description of this type of reentrant phase behavior is given in Ref. [4].

Early theoretical studies of this particular phenomenon of fluid phase equilibria used lattice models with orientationally dependent intermolecular interactions (see Ref. [5] for a review). Such approaches are based on the assumption that a lattice gives an adequate description of the structure of a liquid, which is a significant oversimplification. A description of closed-loop immiscibility obtained with continuum fluid theories is more realistic, since features such as pressure and density can be directly related to those of a real fluid.
The first continuum study of a system with a well-defined directional intermolecular potential which exhibits closedloop immiscibility [5] used the Wertheim approach [6-9] to describe the association interactions within the framework of the statistical associating fluid theory (SAFT) [10-13]. More recently, the link between the existence of the LCST and short-ranged, directional association interactions has been established for a continuum model using computer simulation [14]. Here, we illustrate how continuum fluid theories, more specifically the statistical associating fluid theory as adapted to potentials of variable range (SAFT-VR) $[15,16]$, can be used to give complementary results in a study of the effect of pressure and association interaction strength on the phase diagram of a simple model system which exhibits reentrant miscibility.

It is well known that continuum fluid theories such as SAFT can be applied to obtain an accurate prediction of the phase behavior of real systems, providing suitable models are used. The seminal study of Scott and van Konynenburg $[17,18]$ used the van der Waals equation of state to describe, and hence classify, the types of phase behavior exhibited by binary fluid mixtures. Type VI behavior, which incorporates regions of closed-loop immiscibility, is the only type which cannot be predicted theoretically with the van der Waals equation of state. The SAFT methodology consists of a perturbation theory about a hard-sphere reference system, where 
the dispersive interactions are given by a simple hightemperature expansion, and a term is included to account for the association of species. The extent of the closed-loop region in the model system studied in Ref. [5] with a simplified (mean-field) SAFT equation of state depends on both the pressure of the system and the strength of the short-ranged interaction between species in the mixture. A global study of the phase behavior of aqueous mixtures of alcohols using the SAFT approach predicts the existence of closed-loop regions of immiscibility for model associating systems of intermediate chain lengths with specific values of the site-site interaction energy [19]. Similar models were used to give an accurate prediction of the phase equilibria of aqueous systems of 2-butanol, butoxyethanol and long-chain nonionic alkyl polyoxyethylene surfactant molecules $\left(C_{i} E_{j}\right)$ with the SAFT approach [20,21]. This simple approach is shown to give an adequate description of the phase behavior of systems where association is the dominant contribution to the Helmholtz free energy.

A recent extension of the SAFT methodology employs the Barker and Henderson high-temperature perturbation theory [22-24] to give a more accurate description of the dispersive interactions, and can also be used to predict the phase behavior of systems which interact via potentials of variable range. This so-called SAFT-VR approach $[15,16]$ is described in more detail in Sec. III. A major advantage of the SAFT approach is that only the monomer Helmholtz free energy and the contact value of the monomer cavity function are required to describe the equation of state of chain molecules. The success of this approach lies in its ability to provide an accurate prediction of the thermodynamics and hence the phase behavior of systems where molecular shape and associating phenomena are dominant features. Additionally, the SAFT approach is very versatile; it can be used to describe the phase behavior of a number of different systems (see Ref. [15] for a brief review). The molecular based nature of the SAFT-VR equation allows the results to be compared with computer simulation.

Here we obtain the phase behavior of a symmetrical model mixture using both the Gibbs ensemble Monte Carlo simulation method and the SAFT-VR equation of state. The system consists of a binary mixture of equal sized spheres with unfavorable mean-field interactions (which lead to liquid-liquid phase separation at intermediate temperatures) and a single association site which provides the directional interactions between unlike species (which leads to miscibility at low temperatures). The particles interact with a squarewell potential:

$$
u_{i j}\left(r_{i j}\right)=\left\{\begin{array}{cc}
+\infty & \text { if } r_{i j}<\sigma_{i j}, \\
-\varepsilon_{i j} & \text { if } \sigma_{i j} \leqslant r_{i j}<\lambda_{i j} \sigma_{i j}, \\
0 & \text { if } r_{i j} \geqslant \lambda_{i j} \sigma_{i j},
\end{array}\right.
$$

where $r_{i j}$ is the distance between two particles. The contact distance is $\sigma_{i j}$ and the parameters $\lambda_{i j}$ and $\varepsilon_{i j}$ are the range and depth of the potential well for the $i-j$ interaction, respectively. For our particular system $\sigma=\sigma_{11}=\sigma_{12}=\sigma_{22}, \quad \varepsilon$ $=\varepsilon_{11}=\varepsilon_{22}$, and $\varepsilon_{12}=0$, with $\lambda=\lambda_{11}=\lambda_{12}=\lambda_{22}=1.5$. A single square-well association site of fixed depth $\varepsilon_{a, b}$ and width $\lambda_{a, b}=0.55$ is placed at a distance $r_{d}=0.25 \sigma$ from the center of each sphere. Bonding is only permitted between unlike species, i.e., between site $a$ on component 1 and site $b$ on component 2. This particular model system is known from previous studies to to exhibit regions of closed-loop immiscibility $[5,14]$. A series of simulations are performed for different values of the pressure and site-site interaction energies in order to illustrate their effect on the phase diagram. The results obtained are compared with those obtained using the SAFT-VR approach for corresponding conditions.

\section{GIBBS ENSEMBLE MONTE CARLO SIMULATIONS}

The Gibbs ensemble Monte Carlo (GEMC) method $[25,26]$ is the most common direct simulation technique used for the determination of phase equilibria in fluid systems, particularly in mixtures $[27,28]$. The isothermal-isobaric $(N P T)$ version of the technique consists of a separate simulation in two regions $a$ and $b$ which are in thermodynamic equilibrium but not in physical contact. These regions have volumes $V^{a}$ and $V^{b}$ and contain $N^{a}$ and $N^{b}$ particles, such that $V=V^{a}+V^{b}$ and $N=N^{a}+N^{b}$. The two regions are in thermodynamic equilibrium but not in physical contact. Three different Monte Carlo moves are performed in order to satisfy the conditions of thermodynamic equilibrium: particle displacements and reorientations within either subsystem, to maintain equality of temperature; volume changes of either subsystem, to maintain equality of pressure; and particle interchanges between the two subsystems, to maintain equality of chemical potential. The energy $E^{j}$, volume $V^{j}$, and composition $x_{i}^{j}=N_{i}^{j} / N^{j}$ of particles of type $i$ in subsystem $j$ thus vary during the course of the simulation. The acceptance criterion for each of these moves in the NPT version of the Gibbs ensemble is governed by the pseudo-Boltzmann probability distribution, see Refs. [26,29] for details:

$$
\begin{aligned}
\mathcal{P}^{\text {Gibbs }}= & \exp \left[\ln \left(\frac{N_{1} !}{N_{1}^{a} ! N_{1}^{b} !}\right)+\ln \left(\frac{N_{2} !}{N_{2}^{a} ! N_{2}^{b} !}\right)+N^{a} \ln V^{a}\right. \\
& \left.+N^{b} \ln V^{b}-\frac{P V^{a}}{k T}-\frac{P V^{b}}{k T}-\frac{E^{a}\left(N^{a}\right)}{k T}-\frac{E^{b}\left(N^{b}\right)}{k T}\right] .
\end{aligned}
$$

We examine a symmetrical binary mixture of $N=N_{1}+N_{2}$ particles at a constant temperature $T$ and a constant pressure $P$.

Simulations are performed in cubic boxes with the particles initially arranged on a face-centered-cubic (fcc) lattice. The usual periodic boundary conditions and minimum image convention are used [30]. Initial guesses for the coexisting densities and compositions at each pressure and temperature are made by using the corresponding SAFT-VR solutions (see following section); it is important to ensure that the overall composition of the system lies somewhere between the compositions of the two coexisting phases. The chemical potential is determined with the Widom test particle technique [31] as adapted to the GEMC approach [32], in order to ensure that phase equilibria is achieved. One simulation cycle consists of $N$ displacements and reorientations in each box, one volume change for either box, and a specific number of particle interchanges. The maximum displacement and volume change are adjusted to give an acceptance ratio of 
between 30 and $40 \%$, and the number of insertions is controlled so that between 1 and $3 \%$ of particles are interchanged each cycle. Simulations are performed with systems of $N=512, N=1000$, and $N=1728$ particles at a series of coexistence pressures for different values of the site-site interaction energy. An initial simulation of 50000 cycles is performed to equilibrate the subsystems, before averaging for between 100000 and 250000 cycles.

\section{SAFT-VR EQUATION OF STATE FOR SQUARE- WELL MIXTURES}

The SAFT-VR equation of state for a mixture of associating chain molecules is written in terms of four separate contributions to the Helmholtz free energy $[15,16]$

$$
\frac{A}{N k T}=\frac{A^{\mathrm{IDEAL}}}{N k T}+\frac{A^{\mathrm{MONO}}}{N k T}+\frac{A^{\mathrm{CHAIN}}}{N k T}+\frac{A^{\mathrm{ASSOC}}}{N k T},
$$

where $N$ is the number of chain molecules in the mixture, $k$ is Boltzmann's constant, and $T$ is the temperature. In this equation $A^{\mathrm{IDEAL}}$ is the ideal free energy, $A^{\mathrm{MONO}}$ is the residual free energy due to the monomer segments, $A^{\text {CHAIN }}$ is the residual contribution due to the formation of chains of monomers, and $A^{\mathrm{ASSOC}}$ is the residual contribution to the free energy due to intermolecular association via sites placed on the monomer segments. We present the general expressions for each contribution to the Helmholtz free energy in the above equation together with those which are specific for the associating system examined in this work.

The free energy of an ideal $n$-component mixture is given by [33]

$$
\frac{A^{\mathrm{IDEAL}}}{N k T}=\sum_{i=1}^{n} x_{i} \ln \rho_{i} \Lambda_{i}-1=x_{1} \ln \rho_{1} \Lambda_{1}^{3}+x_{2} \ln \rho_{2} \Lambda_{2}^{3}-1,
$$

where $x_{i}=N_{i} / N$ is the mole fraction, $\rho_{i}=N_{i} / V$ is the number density, and $\Lambda_{i}$ is the thermal de Broglie wavelength of species $i$.

The monomer free energy is

$$
\frac{A^{\mathrm{MONO}}}{N k T}=\left(\sum_{i=1}^{n} x_{i} m_{i}\right) \frac{A^{M}}{N_{s} k T}=\left(\sum_{i=1}^{n} x_{i} m_{i}\right) a^{M}=a^{M},
$$

where $m_{i}$ is the number of spherical segments in each chain $i$, so that $m=1$ for monomers, and $N_{s}$ is the total number of segments. The monomer free energy per segment of the mixture $a^{M}=A^{M} /\left(N_{s} k T\right)$ is obtained from the Barker and Henderson high-temperature expansion [22-24]:

$$
a^{M}=a^{\mathrm{HS}}+\beta a_{1}+\beta^{2} a_{2},
$$

where $a^{\mathrm{HS}}$ is the free energy for a mixture of hard spheres, $\beta=1 / k T, a_{1}$ and $a_{2}$ are the first two perturbation terms associated with the attractive energy.

The free energy of the reference hard-sphere mixture is obtained from the expression of Boublík [34] and Mansoori et al. [35]:

$$
a^{\mathrm{HS}}=\frac{6}{\pi \rho_{s}}\left[\left(\frac{\zeta_{2}^{3}}{\zeta_{3}^{2}}-\zeta_{0}\right) \ln \left(1-\zeta_{3}\right)+\frac{3 \zeta_{1} \zeta_{2}}{1-\zeta_{3}}+\frac{\zeta_{2}^{3}}{\zeta_{3}\left(1-\zeta_{3}\right)^{2}}\right]
$$

In this expression $\rho_{s}=N_{s} / V$ is the number density of the mixture in terms of the number of spherical segments. Note that in general $\rho_{s}=\rho\left(\sum_{i} x_{i} m_{i}\right)$, where $\rho$ is the total number density of the mixture. In this case, since we consider only monatomic species $\rho_{s}=\rho$. The reduced densities $\zeta_{l}$ are defined as

$$
\zeta_{l}=\frac{\pi}{6} \rho_{s}\left[\sum_{i=1}^{n} x_{s, i}\left(\sigma_{i}\right)^{l}\right]
$$

where $\sigma_{i}$ is the diameter of spherical segments of chain $i$, and $x_{s, i}$ is the mole fraction of segments of type $i$ in the mixture, which is given by

$$
x_{s, i}=\frac{m_{i} x_{i}}{\sum_{k=1}^{n} m_{k} x_{k}} .
$$

Since $x_{s, i}=x_{i}$ and $\sigma=\sigma_{1}=\sigma_{2}$ in the system studied here, the free energy of the reference hard-sphere mixture reduces to the Carnahan and Starling expression [36,33]

$$
a^{\mathrm{HS}}=\frac{4 \eta-3 \eta^{2}}{(1-\eta)^{2}}
$$

where $\eta=\pi \rho \sigma^{3} / 6$ is the packing fraction of the pure component, which is equivalent to $\zeta_{3}$ in Eq. (8).

The mean-attractive energy $a_{1}$ in the perturbation expansion is given by

$$
a_{1}=\sum_{i=1}^{n} \sum_{j=1}^{n} x_{s, i} x_{s, j} a_{1}^{i j}
$$

where

$$
a_{1}^{i j}=-2 \pi \rho_{s} \varepsilon_{i j} \int_{\sigma_{i j}}^{\infty} r_{i j}^{2} g_{i j}^{\mathrm{HS}}\left(r_{i j} ; \zeta_{3}\right) d r_{i j},
$$

and $g_{i j}^{\mathrm{HS}}$ is the radial distribution function for a mixture of hard spheres. The integral is transformed by applying the mean-value theorem [15] giving an expression for $a_{1}$ in terms of the contact value of $g_{i j}^{\mathrm{HS}}$ :

$$
a_{1}=-\rho_{s} \sum_{i=1}^{n} \sum_{j=1}^{n} x_{s, i} x_{s, j} \alpha_{i j}^{\mathrm{VDW}} g_{i j}^{\mathrm{HS}}\left[\sigma_{i j} ; \zeta_{3}^{\text {eff }}\right] \text {, }
$$

where

$$
\alpha_{i j}^{\mathrm{VDW}}=2 \pi \varepsilon_{i j} \sigma_{i j}^{3}\left(\lambda_{i j}^{3}-1\right) / 3
$$

is the van der Waals attractive constant for the square-well interaction $i-j$. The contact value of the radial distribution function for the hard-sphere reference system, $g_{i j}^{\mathrm{HS}}\left[\sigma_{i j} ; \zeta_{3}^{\text {eff }}\right]$ is evaluated at an effective packing fraction $\zeta^{\text {eff }}$, using the expression of Boublík [34] 


$$
\begin{aligned}
g_{i j}^{\mathrm{HS}}\left[\sigma_{i j} ; \zeta_{3}^{\mathrm{eff}}\right]= & \frac{1}{1-\zeta_{3}^{\mathrm{eff}}}+3 \frac{\sigma_{i i} \sigma_{j j}}{\sigma_{i i}+\sigma_{j j}} \frac{\zeta_{2}^{\text {eff }}}{\left(1-\zeta_{3}^{\text {eff }}\right)^{2}} \\
& +2\left(\frac{\sigma_{i i} \sigma_{j j}}{\sigma_{i i}+\sigma_{j j}}\right)^{2} \frac{\zeta_{2}^{\mathrm{eff}^{2}}}{\left(1-\zeta_{3}^{\text {eff }}\right)^{3}} .
\end{aligned}
$$

For the binary mixture studied here $\sigma=\sigma_{11}=\sigma_{22}, \varepsilon=\varepsilon_{11}$ $=\varepsilon_{22}, \lambda=\lambda_{11}=\lambda_{12}=\lambda_{22}$, and $\varepsilon_{12}=0$, so that the meanattractive energy of Eq. (11) reduces to

$$
a_{1}=x_{1}^{2} a_{1}^{11}+x_{2}^{2} a_{1}^{22}=\left(x_{1}^{2}+x_{2}^{2}\right) a_{1}^{\mathrm{SW}},
$$

where

$$
a_{1}^{\mathrm{SW}}=-\rho_{s} \alpha^{\mathrm{VDW}} g^{\mathrm{HS}}\left[\sigma ; \eta^{\mathrm{eff}}\right],
$$

with

$$
\alpha^{\mathrm{VDW}}=2 \pi \varepsilon \sigma^{3}\left(\lambda^{3}-1\right) / 3
$$

The Carnahan and Starling equation for the contact value of the pair correlation function is used [36,33],

$$
g^{\mathrm{HS}}\left[\sigma ; \eta^{\mathrm{eff}}\right]=\frac{1-\eta^{\mathrm{eff}} / 2}{\left(1-\eta^{\mathrm{eff}}\right)^{3}},
$$

with $\eta^{\text {eff }}=\zeta_{3}^{\text {eff }}$. The parameterization for $\eta^{\text {eff }}$ obtained for the pure square-well fluid [15] is used, where

$$
\eta^{\mathrm{eff}}(\eta, \lambda)=c_{1}(\lambda) \eta+c_{2}(\lambda) \eta^{2}+c_{3}(\lambda) \eta^{3}
$$

and the coefficients $c_{1}, c_{2}$, and $c_{3}$ are given by

$$
\begin{aligned}
\left(\begin{array}{l}
c_{1} \\
c_{2} \\
c_{3}
\end{array}\right)= & \left(\begin{array}{ccc}
2.25855 & -1.50349 & 0.249434 \\
-0.669270 & 1.40049 & -0.827739 \\
10.1576 & -15.0427 & 5.30827
\end{array}\right) \\
& \times\left(\begin{array}{c}
1 \\
\lambda \\
\lambda^{2}
\end{array}\right) .
\end{aligned}
$$

This corresponds to the MX1 or MX3 mixing rules of Ref. [16].

The fluctuation term of the free energy is given by

$$
a_{2}=\sum_{i=1}^{n} \sum_{j=1}^{n} x_{s, i} x_{s, j} a_{2}^{i j},
$$

and each of the terms $a_{2}^{i j}$ are obtained with the local compressibility approximation (LCA) [22,23],

$$
a_{2}^{i j}=\frac{1}{2} K^{\mathrm{HS}} \varepsilon_{i j} \rho_{s} \frac{\partial a_{1}^{i j}}{\partial \rho_{s}},
$$

where $K^{\mathrm{HS}}$ is the isothermal compressibility for a mixture of hard spheres which is given by the Percus-Yevick expression [37]

$$
K^{\mathrm{HS}}=\frac{\zeta_{0}\left(1-\zeta_{3}\right)^{4}}{\zeta_{0}\left(1-\zeta_{3}\right)^{2}+6 \zeta_{1} \zeta_{2}\left(1-\zeta_{3}\right)+9 \zeta_{2}^{3}} .
$$

For our particular system

$$
a_{2}=x_{1}^{2} a_{2}^{11}+x_{2}^{2} a_{2}^{22}=\left(x_{1}^{2}+x_{2}^{2}\right) a_{2}^{\mathrm{SW}},
$$

with

$$
a_{2}^{\mathrm{SW}}=\frac{1}{2} K^{\mathrm{HS}} \varepsilon \rho_{s} \frac{\partial a_{1}^{\mathrm{SW}}}{\partial \rho_{s}},
$$

where $K^{\mathrm{HS}}$ is now the pure component expression

$$
K^{\mathrm{HS}}=\frac{(1-\eta)^{4}}{(1-\eta)^{2}+6 \eta(1-\eta)+9 \eta^{2}} \text {. }
$$

Since the system examined here consists only of monatomic species the contribution to the free energy in Eq. (3) due to chain formation is zero $\left(A^{\text {CHAIN }}=0\right)$.

Within the framework of the theory of Wertheim the contribution to the free energy in the SAFT-VR equation of state due to the association mediated by $s_{i}$ sites on molecules of species $i$ is described by [11]

$$
\frac{A^{\mathrm{ASSOC}}}{N k T}=\sum_{i=1}^{n} x_{i}\left[\sum_{a=1}^{s_{i}}\left(\ln X_{a, i}-\frac{X_{a, i}}{2}\right)+\frac{s_{i}}{2}\right] \text {. }
$$

The first sum is over the number of species $i$ in the mixture and the second sum is over all sites $a$ on a molecule of type $i$. The fractions of molecules of species $i$ not bonded at a particular site $a, X_{a, i}$, is given by solution of the simultaneous equations:

$$
X_{a, i}=\frac{1}{1+\sum_{j=1}^{n} \sum_{b=1}^{s_{j}} \rho x_{j} X_{b, j} \Delta_{a, b, i, j}},
$$

where

$$
\Delta_{a, b, i, j}=K_{a, b, i, j} f_{a, b, i, j} g_{i j}^{\mathrm{SW}}\left(\sigma_{i j}\right)
$$

is specific for each $a-b$ site-site interaction, and incorporates the volume available for bonding $K_{a, b, i, j}$, and the strength of the association via the Mayer function $f_{a, b, i, j}=\exp \left(\varepsilon_{a b} / k T\right)$ -1 of the square-well potential. The contact value of the radial distribution function for the square-well interaction $g_{i j}^{\mathrm{SW}}\left(\sigma_{i j}\right)$ is evaluated within the SAFT-VR approach for mixtures $[15,16]$ using a first order perturbation expansion

$$
g_{i j}^{\mathrm{SW}}\left(\sigma_{i j}\right)=g_{i j}^{\mathrm{HS}}\left(\sigma_{i j}\right)+\beta \epsilon_{i j} g_{1}\left(\sigma_{i j}\right) .
$$

The contact value of the radial distribution function for the hard-sphere reference system $g_{i j}^{\mathrm{HS}}\left[\sigma_{i j} ; \zeta_{3}\right]$ is evaluated with the actual packing fraction $\zeta_{3}$, using the expression of Eq. (15). The first order perturbation term $g_{1}\left(\sigma_{i j}\right)$ is obtained from a self-consistent calculation of the pressure using the Clausius virial theorem [15]: 


$$
\begin{aligned}
g_{i j}^{\mathrm{SW}}\left[\sigma_{i j} ; \zeta_{3}\right]= & g_{i j}^{\mathrm{HS}}\left[\sigma_{i j} ; \zeta_{3}\right]+\beta \varepsilon_{i j}\left[g_{i j}^{\mathrm{HS}}\left[\sigma_{i j} ; \zeta_{3}^{\mathrm{eff}}\right]+\left(\lambda_{i j}^{3}-1\right)\right. \\
& \left.\times\left(\frac{\lambda_{i j}}{3} \frac{\partial g_{i j}^{\mathrm{HS}}\left[\sigma_{i j} ; \zeta_{3}^{\mathrm{eff}}\right]}{\partial \lambda_{i j}}-\rho_{s} \frac{\partial g_{i j}^{\mathrm{HS}}\left[\sigma_{i j} ; \zeta_{3}^{\mathrm{eff}}\right]}{\partial \rho_{s}}\right)\right] .
\end{aligned}
$$

For our system, the contact value of the square-well distribution function of Eq. (32) simplifies to

$$
\begin{aligned}
g^{\mathrm{SW}}[\sigma ; \eta]= & g^{\mathrm{HS}}[\sigma ; \eta]+\beta \varepsilon\left[g^{\mathrm{HS}}\left[\sigma ; \eta^{\mathrm{eff}}\right]+\left(\lambda^{3}-1\right)\right. \\
& \left.\times \frac{\partial g^{\mathrm{HS}}\left[\sigma ; \eta^{\mathrm{eff}}\right]}{\partial \eta^{\mathrm{eff}}}\left(\frac{\lambda}{3} \frac{\partial \eta^{\mathrm{eff}}}{\partial \lambda}-\eta \frac{\partial \eta^{\mathrm{eff}}}{\partial \eta}\right)\right],
\end{aligned}
$$

where the Carnahan and Starling expressions [36,33] for $g^{\mathrm{HS}}[\sigma ; \eta]$ and $g^{\mathrm{HS}}\left[\sigma ; \eta^{\text {eff }}\right]$ are used with the total and effective packing fractions, respectively. The parametrization for $\eta^{\text {eff }}$ for the pure square-well fluid is given in Eqs. (20) and (21).

The components in the mixture examined here have only have one interaction site per sphere and bonding is only permitted between unlike components in the mixture, i.e., between site $a$ on component 1 and site $b$ on component 2 when the two sites are within a distance $r_{c}$. The expression for the contribution to the Helmholtz free energy due to association, Eq. (28), thus simplifies to

$$
\frac{A^{\mathrm{ASSOC}}}{N k T}=x_{1}\left(\ln X_{a}-\frac{X_{a}}{2}+\frac{1}{2}\right)+x_{2}\left(\ln X_{b}-\frac{X_{b}}{2}+\frac{1}{2}\right) \text {. }
$$

The fractions $X_{a}$ and $X_{b}$ of molecules of species 1 and 2 not bonded at sites $a$ and $b$, respectively, are given by

$$
X_{a}=\frac{1}{1+\rho x_{2} X_{b} \Delta_{a, b}}
$$

and

$$
X_{b}=\frac{1}{1+\rho x_{1} X_{a} \Delta_{a, b}},
$$

where

$$
\Delta_{a, b}=K_{a, b} f_{a, b} g^{\mathrm{SW}}(\sigma) .
$$

The volume available for bonding between two sites $a$ and $b$ which are positioned at a distance $r_{d}$ from the center of spheres with diameter $\sigma$ and have an interaction range $r_{c}$ is given by $[10]$

$$
\begin{aligned}
K_{a, b}= & 4 \pi^{2} \sigma\left\{\ln \left[\left(r_{c}+2 r_{d}\right) / \sigma\right]\left(6 r_{c}^{3}+18 r_{c}^{2} r_{d}-24 r_{d}^{3}\right)\right. \\
& +\left(r_{c}+2 r_{d}-\sigma\right)\left(22 r_{d}^{2}-5 r_{c} r_{d}-7 r_{d} \sigma-8 r_{c}^{2}+r_{c} \sigma\right. \\
& \left.\left.+\sigma^{2}\right)\right\} /\left(72 r_{d}^{2}\right) .
\end{aligned}
$$

In order to determine the conditions of phase equilibria for the model system, the pressure and chemical potential must be obtained from the expression for the Helmholtz free energy. The chemical potential $\mu_{i}$ of species $i$ is given by

$$
\frac{\mu_{i}}{k T}=\left(\frac{\partial A / k T}{\partial N_{i}}\right)_{T, V, N_{j \neq i}}
$$

where $N_{i}$ is the number of molecules of species $i$. The pressure $P$ may be calculated through the compressibility factor $Z$ as

$$
Z=\frac{P V}{N k T}=\sum_{i=1}^{n} x_{i} \frac{\mu_{i}}{k T}+\frac{A}{N k T}
$$

These functions are used in the numerical determination of the phase behavior of the mixture, using a simplex method [38].

\section{CRITICAL BEHAVIOR}

Generally, finite size effects in the Gibbs ensemble are considered to be small for regions away from the critical point [39], so that simulation data from these regions can be used as experimental data to estimate the critical temperature $T_{c}(N)$ via extrapolation of a Wegner expansion [40] which includes scaling corrections [41]. This method has been used successfully to predict the critical parameters of the pure Lennard-Jones fluid [42], the pure square-well fluid [43], and selected square-well mixtures [44]. Before such a fitting procedure can be performed it is necessary to identify the universality class [45] of the phase transitions occurring at the UCST and the LCST for the symmetrical associating mixture. As the critical point between two phases $a$ and $b$ is approached the critical exponent $\beta$ is defined in terms of the critical temperature of the finite system as [46]

$$
\Delta x(N)=\left|x_{a}-x_{b}\right|=B_{0}(N)\left|1-\frac{T}{T_{c}(N)}\right|^{\beta},
$$

where $B_{0}(N)$ is the leading amplitude term. We assume that the phase transitions of the binary mixture at the LCST and the UCST both belong to the Ising universality class, so that the critical exponent has the universal value of $\beta=0.325$. The coexistence curve thus has a cubic form in the critical region. Providing that the correlation length $\zeta_{a}$ remains less than the linear box length $L$, the value of $\beta=0.325$ can be assumed to be valid for the duration of the simulations. A change in the value of the critical exponent from $\beta=0.325$ (universal) to $\beta=0.5$ (mean-field) only occurs when the correlation length becomes greater than $L$ [47]. A value of $\beta$ $=0.5$ results in a quadratic coexistence curve close to the critical temperature. This "crossover" of critical exponents has only been observed in Gibbs ensemble simulation studies of a two-dimensional lattice gas [47] and in a two dimensional square-well mixture [48]. Simulation studies of the square-well fluid in three dimensions $[48,44,49]$, show no sign of this crossover behavior. This is in agreement with the opinion that this type of crossover cannot be observed in three dimensional systems using regular simulation techniques [50].

Away from the critical region the power law of Eq. (41) fails to accurately describe the shape of the coexistence 
curve. Corrections to the renormaliZation-group (RG)-based scaling laws must be included in order to obtain more accurate results [41]. Such corrections can be written as an expansion in $t=1-T / T_{c}(N)$, in a so-called Wegner [40] expansion

$$
\left|x_{a}-x_{b}\right|=B_{0}|t|^{\beta}+B_{1}|t|^{\beta+\Delta_{1}}+B_{2}|t|^{\beta+2 \Delta_{1}}+\cdots,
$$

where $\Delta_{1}=0.5$ is a RG gap exponent, and the $B_{i}$ terms are the correction amplitudes. This expansion leads an estimate of the critical temperature while assuming a universal value for the critical exponent. A similar expansion can be written for the diameter of the coexistence curve [41]

$$
\frac{\left(x_{a}+x_{b}\right)}{2}=x_{c}+C_{1}|t|^{\psi}+C_{2}|t|+C_{3}|t|^{\psi+\Delta_{1}}+\cdots,
$$

where $x_{c}$ is the critical composition, the $C_{i}$ terms are the coefficients of the expansion, and $\psi$ is an exponent which characterizes the anomaly in the diameter of the curve. These two expansions can be combined to give one expression for the coexisting compositions

$$
\begin{aligned}
x_{ \pm}= & x_{c}+C_{1}|t|^{\psi}+C_{2}|t|+C_{3}|t|^{\psi+\Delta_{1}}+\cdots \\
& \pm \frac{1}{2}\left(B_{0}|t|^{\beta}+B_{1}|t|^{\beta+\Delta_{1}}+B_{2}|t|^{\beta+2 \Delta_{1}}+\cdots\right),
\end{aligned}
$$

where $x_{-}$and $x_{+}$represent the smaller and larger coexistence compositions, respectively. Similar expressions can be written in terms of the coexistence densities.

It is important to note that the Wegner extended scaling of the GEMC simulation data for simple model systems such as the square-well fluid provides estimates for the critical parameters with an equivalent accuracy to the more thorough analysis of the critical region obtained by using finite-size scaling approaches (see Refs. [44,48,51]), despite being significantly less computationally demanding.

For the associating square-well mixture studied here this expression can be simplified due to the symmetry of the system. The critical composition is known, $x_{c}=0.5$, and the $C_{i}$ coefficients can be disregarded, since the diameter is symmetrical about $x_{c}=0.5$. Additionally, inclusion of only the first correction to scaling term $B_{1}$ is known to give an adequate description of the liquid-liquid coexistence region [44]. The expression for the compositions of the coexisting phases becomes

$$
x_{ \pm}=\frac{1}{2} \pm \frac{1}{2}\left(B_{0}|t|^{\beta}+B_{1}|t|^{\beta+\Delta_{1}}\right) \text {. }
$$

Assuming values of $\beta=0.325$ and $\Delta_{1}=0.5$ the above equation can be fitted to the simulation data using a standard least-squares procedure [38] to give estimates of the critical temperature. For coexistence curves which possess both an upper and a lower critical solution temperature it is possible to use two such power laws, one to estimate the UCST, and one to estimate the LCST. These two expressions can be combined to give a complete description of the closed-loop coexistence curve. A hyperbolic tangent

$$
\tanh (y)=\frac{\exp (y)-\exp (-y)}{\exp (y)+\exp (-y)}
$$

is used as a switching function between the expansion for the UCST and that for the LCST. Hence, the expansion fitted to data in the region of the UCST is used above a certain temperature $T_{\text {switch }}$, and the expansion fitted to data in the region of the LCST is used at temperatures below this point. The compositions of the coexisting liquid phases in the closedloop region are obtained as

$$
x_{ \pm}^{\text {loop }}=F(T) x_{ \pm}^{\mathrm{UCST}}+(1-F(T)) x_{ \pm}^{\mathrm{LCST}},
$$

where $F(T)$ is given by

$$
F(T)=\frac{1}{2}+\frac{1}{2} \tanh (y),
$$

with

$$
y=\frac{T-T_{\text {switch }}}{2 \lambda_{\text {switch }}} .
$$

The parameter $\lambda_{\text {switch }}$ controls the steepness of the switching function.

\section{RESULTS}

The reduced thermodynamic variables, temperature $T^{*}=k T / \varepsilon$, pressure $P^{*}=P \sigma^{3} / \varepsilon$, and site-site interaction energy $\varepsilon_{a, b}^{*}=\varepsilon_{a, b} / \varepsilon$ are used in the following discussion. It is also convenient to reduce the pressure and temperature with respect to the critical point of one of the components

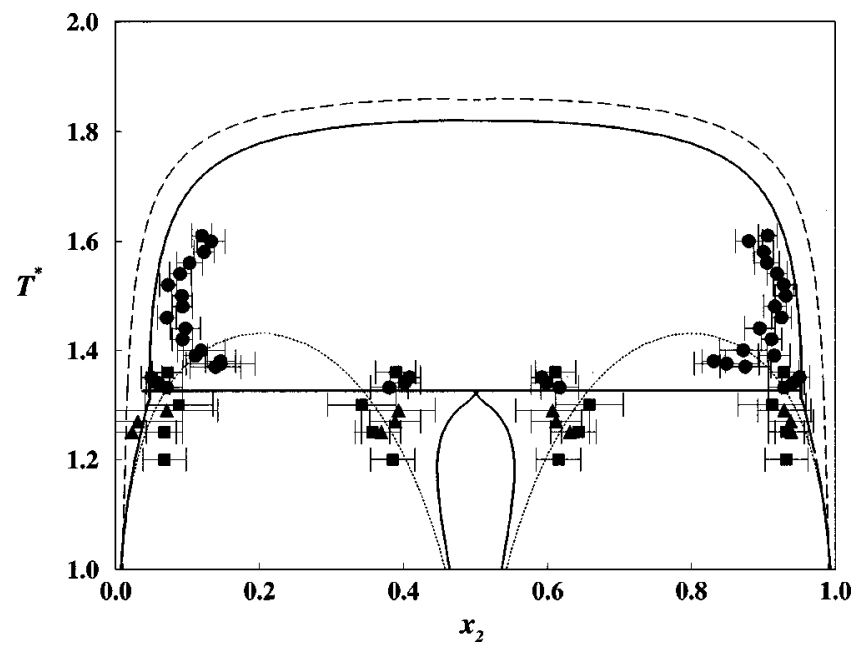

FIG. 1. Temperature-composition slice of the coexistence region for the symmetrical square-well mixture at a reduced pressure of $P^{*}=P \sigma^{3} / \varepsilon=0.756$ with a bonding interaction of $\varepsilon_{a, b}^{*}=\varepsilon_{a, b} / \varepsilon$ $=13$. The reduced temperature is defined as $T^{*}=k T / \varepsilon$. The triangles correspond to the GEMC data for a system of $N=512$ particles, the squares to a system of $N=1000$ particles, and the circles to a system of $N=1728$ particles. The error bars correspond to one standard deviation. The dashed, continuous, and dotted curves correspond to the SAFT-VR prediction for the same model with bonding strengths of $\varepsilon_{a, b}^{*}=12,13$, and 14 , respectively. The predicted three-phase line is represented by the horizontal line. 


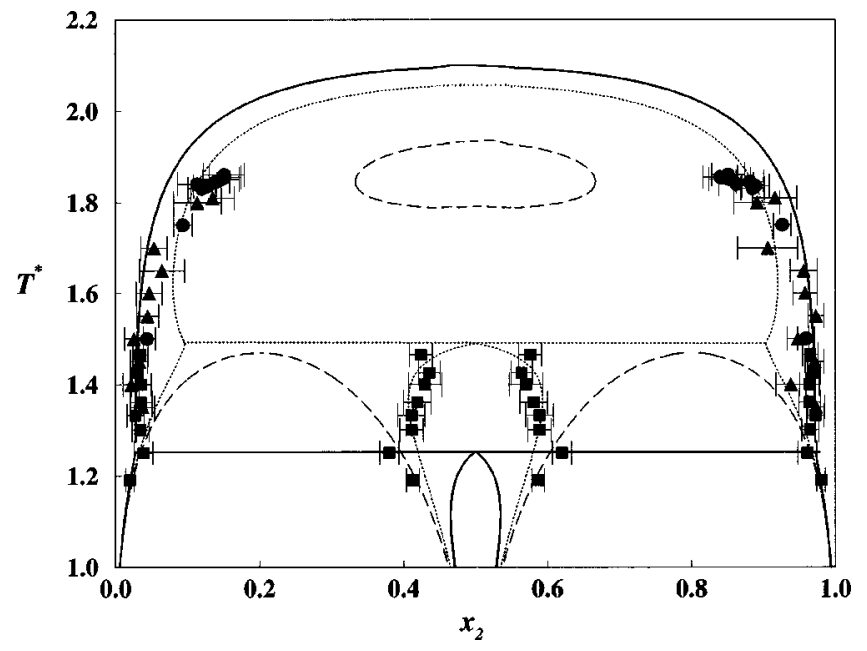

FIG. 2. Temperature-composition slice of the coexistence region for the symmetrical square-well mixture at a reduced pressure of $P^{*}=P \sigma^{3} / \varepsilon=1.08$ with a bonding interaction of $\varepsilon_{a, b}^{*}=\varepsilon_{a, b} / \varepsilon$ $=13$. The reduced temperature is defined as $T^{*}=k T / \varepsilon$. The triangles correspond to the GEMC data for a system of $N=512$ particles, the squares to a system of $N=1000$ particles, and the circles to a system of $N=1728$ particles. The error bars correspond to one standard deviation. The continuous, dotted, and dashed curves correspond to the SAFT-VR prediction for the same model with bonding strengths of $\varepsilon_{a, b}^{*}=13,14$, and 15 , respectively. The predicted three-phase lines are represented by the horizontal lines.

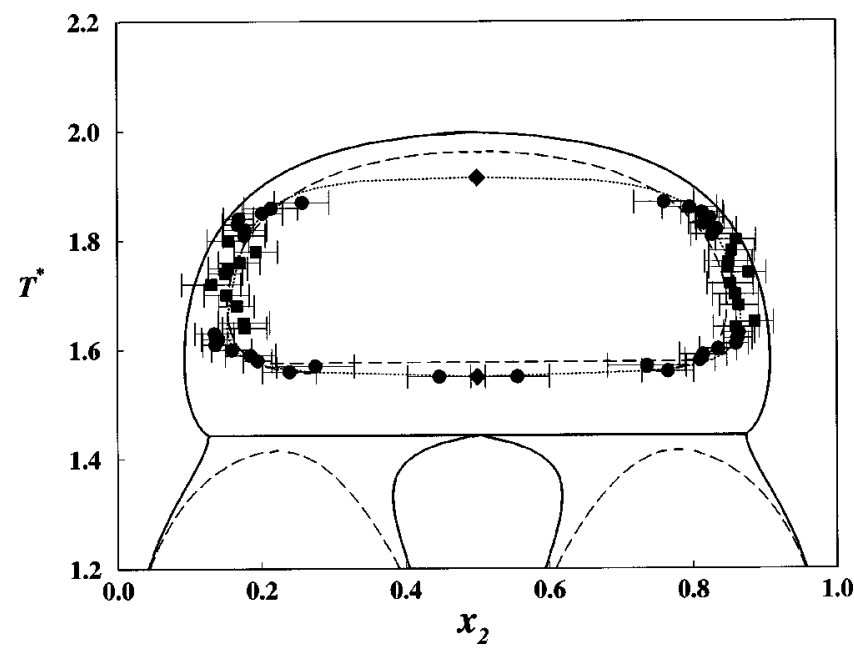

FIG. 3. Temperature-composition slice of the coexistence region for the symmetrical square-well mixture at a reduced pressure of $P^{*}=P \sigma^{3} / \varepsilon=1.28$ with a bonding interaction of $\varepsilon_{a, b}^{*}=\varepsilon_{a, b} / \varepsilon$ $=14.5$. The reduced temperature is defined as $T^{*}=k T / \varepsilon$. The squares correspond to the GEMC data for a system of $N=1000$ particles, and the circles to a system of $N=1728$ particles. The error bars correspond to one standard deviation. The continuous and dashed curves correspond to the SAFT-VR prediction for the same model with bonding strengths of $\varepsilon_{a, b}^{*}=14.5$ and 15 , respectively. The dotted curve corresponds to the fit of the GEMC simulation results obtained using the Wegner expansion of Eq. (47), which includes a first correction to scaling. The predicted three-phase line is represented by the horizontal line.

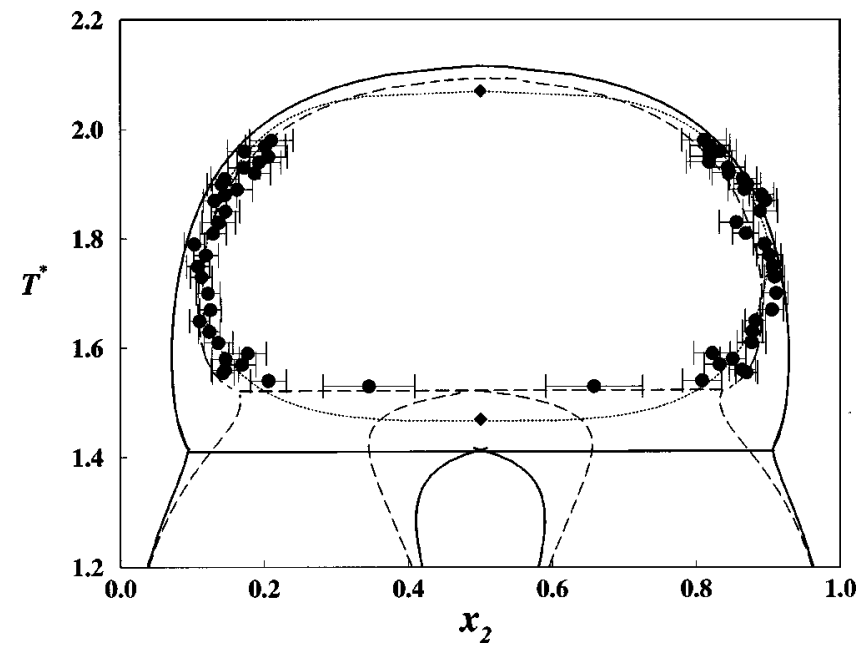

FIG. 4. Temperature-composition slice of the coexistence region for the symmetrical square-well mixture at a reduced pressure of $P^{*}=P \sigma^{3} / \varepsilon=1.48$ with a bonding interaction of $\varepsilon_{a, b}^{*}=\varepsilon_{a, b} / \varepsilon$ $=14.5$. The reduced temperature is defined as $T^{*}=k T / \varepsilon$. The circles correspond to the GEMC data for a system of $N=1728$ particles. The error bars correspond to one standard deviation. The continuous and dashed curves correspond to the SAFT-VR prediction for the same model with bonding strengths of $\varepsilon_{a, b}^{*}=14.5$ and 15 , respectively. The dotted curve corresponds to the fit of the GEMC simulation results obtained using the Wegner expansion of Eq. (47), which includes a first correction to scaling. The predicted three-phase lines are represented by the horizontal lines.

such that $T_{r}=T^{*} / T_{c}^{*}$ and $P_{r}=P^{*} / P_{c}^{*}$, where the subscript $c$ denotes the critical value of a variable. We use the critical values of $T_{c}^{*}=1.219 \pm 0.008$ and $P_{c}^{*}=0.108 \pm 0.016$ for the pure-component square-well system with range $\lambda=1.5 \mathrm{ob}-$ tained from GEMC simulation results [43].

Constant-pressure temperature-composition $T x$ slices of the coexistence regions are shown in Figs. 1-4. The symmetry of the phase diagrams about $x_{2}=0.5$ is a result of the symmetry of the model system. The distinction between gas and liquid fluid phases is arbitrary at high pressures and temperatures; here we use the term "gas" to describe a phase which has its origins in the vapor phase of the pure component and which thus has the lower density.

The pressure of $P^{*}=0.756$ of Fig. 1 is approximately seven times the critical pressure of the pure square-well system with $\lambda=1.5$. The Gibbs ensemble simulation data with $\varepsilon_{a, b}^{*}=13$ for three system sizes are shown; there is a negligible system size effect. The region of liquid-liquid immiscibility bounded by an UCST at high temperatures is clearly shown, together with low temperature regions of gas-liquid immiscibility below a three-phase line. The curves represent the SAFT-VR prediction for the equivalent model, with three different strengths of the site-site interaction energy. For a value of $\varepsilon_{a, b}^{*}=12$ the theory predicts immiscibility of the system at all temperatures below an USCT. As the site-site interaction is increased to $\varepsilon_{a, b}^{*}=13$ the prediction includes regions of gas-liquid immiscibility at lower temperatures, and the three-phase line can be observed at a temperature close to the critical temperature of the pure component. Increasing the strength of the bonding interaction further to $\varepsilon_{a, b}^{*}=14$, leads to a complete change in the nature of the $T x$ 
TABLE I. Liquid-liquid coexistence data obtained from NPT Gibbs-ensemble Monte Carlo simulations for the symmetrical mixture of square wells with a range $\lambda=1.5$, and a single interaction site of reduced depth $\varepsilon_{a, b}^{*}=\varepsilon_{a, b} / \varepsilon=14.5$. The fixed variables during the simulation are the number of particles $N$, the reduced pressure $P^{*}=P \sigma^{3} / \varepsilon=1.28$ and the reduced temperature $T^{*}=k T / \varepsilon$. The packing fractions $\eta$ and mole fractions $x_{2}$ in the coexisting liquid phases are labeled $l_{1}$ and $l_{2}$, respectively; the uncertainties correspond to one standard deviation.

\begin{tabular}{cccccc}
\hline \hline $\mathrm{N}$ & $T^{*}$ & $\eta_{l_{1}}$ & $\eta_{l_{2}}$ & $x_{2, l_{1}}$ & $x_{2, l_{2}}$ \\
\hline 1728 & 1.58 & $0.289 \pm 0.004$ & $0.289 \pm 0.004$ & $0.810 \pm 0.021$ & $0.195 \pm 0.020$ \\
1728 & 1.59 & $0.289 \pm 0.004$ & $0.290 \pm 0.003$ & $0.814 \pm 0.033$ & $0.185 \pm 0.035$ \\
1728 & 1.60 & $0.291 \pm 0.003$ & $0.292 \pm 0.004$ & $0.835 \pm 0.026$ & $0.159 \pm 0.028$ \\
1728 & 1.61 & $0.292 \pm 0.003$ & $0.292 \pm 0.003$ & $0.860 \pm 0.017$ & $0.136 \pm 0.017$ \\
1728 & 1.62 & $0.289 \pm 0.004$ & $0.290 \pm 0.004$ & $0.862 \pm 0.020$ & $0.140 \pm 0.023$ \\
1728 & 1.63 & $0.290 \pm 0.005$ & $0.290 \pm 0.005$ & $0.864 \pm 0.022$ & $0.135 \pm 0.027$ \\
1000 & 1.64 & $0.284 \pm 0.006$ & $0.279 \pm 0.006$ & $0.860 \pm 0.032$ & $0.177 \pm 0.031$ \\
1000 & 1.65 & $0.292 \pm 0.007$ & $0.285 \pm 0.007$ & $0.886 \pm 0.026$ & $0.176 \pm 0.036$ \\
1000 & 1.68 & $0.282 \pm 0.007$ & $0.277 \pm 0.006$ & $0.864 \pm 0.027$ & $0.166 \pm 0.025$ \\
1000 & 1.70 & $0.275 \pm 0.007$ & $0.273 \pm 0.007$ & $0.859 \pm 0.032$ & $0.152 \pm 0.031$ \\
1000 & 1.72 & $0.272 \pm 0.007$ & $0.274 \pm 0.010$ & $0.852 \pm 0.032$ & $0.131 \pm 0.041$ \\
1000 & 1.74 & $0.271 \pm 0.005$ & $0.267 \pm 0.005$ & $0.878 \pm 0.024$ & $0.150 \pm 0.024$ \\
1000 & 1.75 & $0.266 \pm 0.004$ & $0.266 \pm 0.004$ & $0.849 \pm 0.027$ & $0.154 \pm 0.027$ \\
1000 & 1.76 & $0.264 \pm 0.006$ & $0.261 \pm 0.005$ & $0.850 \pm 0.033$ & $0.170 \pm 0.029$ \\
1000 & 1.78 & $0.262 \pm 0.007$ & $0.257 \pm 0.006$ & $0.854 \pm 0.032$ & $0.193 \pm 0.030$ \\
1000 & 1.80 & $0.262 \pm 0.005$ & $0.260 \pm 0.004$ & $0.860 \pm 0.028$ & $0.154 \pm 0.029$ \\
1728 & 1.81 & $0.254 \pm 0.004$ & $0.254 \pm 0.004$ & $0.827 \pm 0.029$ & $0.177 \pm 0.029$ \\
1728 & 1.82 & $0.256 \pm 0.003$ & $0.255 \pm 0.003$ & $0.833 \pm 0.028$ & $0.178 \pm 0.030$ \\
1728 & 1.83 & $0.254 \pm 0.003$ & $0.255 \pm 0.003$ & $0.816 \pm 0.021$ & $0.168 \pm 0.021$ \\
1728 & 1.84 & $0.254 \pm 0.003$ & $0.254 \pm 0.003$ & $0.824 \pm 0.020$ & $0.169 \pm 0.021$ \\
1728 & 1.85 & $0.251 \pm 0.004$ & $0.249 \pm 0.004$ & $0.813 \pm 0.029$ & $0.202 \pm 0.028$ \\
1728 & 1.86 & $0.247 \pm 0.005$ & $0.247 \pm 0.005$ & $0.796 \pm 0.038$ & $0.214 \pm 0.037$ \\
\hline \hline
\end{tabular}

slice, with miscibility above the two low-temperature regions of gas-liquid coexistence. Thus the SAFT-VR equation predicts that an increase in the site-site interaction energy from $\varepsilon_{a, b}^{*}=13$ to $\varepsilon_{a, b}^{*}=14$ is sufficient to bring about the disappearance of the liquid-liquid immiscibility at this pressure. The low-temperature regions of gas-liquid immiscibility obtained from simulation appear at slightly higher temperatures than those predicted by the SAFT-VR equation of state for $\varepsilon_{a, b}^{*}=13$.

The equivalent temperature composition $T x$ slice for a higher pressure of $P^{*}=1.08$ (approximately ten times the critical pressure of the pure component) is shown in Fig. 2. The simulation results again present a region of liquid-liquid immiscibility bounded at high temperatures by an UCST, and low-temperature regions of gas-liquid immiscibility below the three-phase line. The region of liquid-liquid immiscibility is seen to be larger than in the lower pressure case of Fig. 1, but a closed-loop of immiscibility is still not observed for $\varepsilon_{a, b}^{*}=13$. For this value of the site-site interaction energy the SAFT-VR equation of state predicts a region of liquidliquid immiscibility bounded by an UCST and small regions of low temperature gas-liquid coexistence below the three phase line. For a site-site interaction energy of $\varepsilon_{a, b}^{*}=14$, the low temperature gas-liquid regions predicted by the theory are larger than in the $\varepsilon_{a, b}^{*}=13$ case; the onset of a closedloop region can be observed. Upon increasing the association energy to $\varepsilon_{a, b}^{*}=15$ the SAFT-VR equation of state predicts the existence of a small closed-loop of immiscibility bounded at high temperatures by an UCST at $T^{*}=1.98$ and at low temperatures by a LCST at $T^{*}=1.84$, together with low-temperature regions of gas-liquid coexistence.

At this stage we can conclude that increasing the pressure from $P^{*}=0.756$ to $P^{*}=1.08$ for a system with an interaction energy of $\varepsilon_{a, b}^{*}=13$ does not bring about a sufficient change in the phase diagram of the system for a closed-loop of immiscibility to be observed by GEMC simulation. An increase in the strength of the bonding interaction, together with a slight increase in pressure is required in order to observe a closed-loop of immiscibility [14].

The temperature-composition slice for a pressure of $P^{*}=1.28$ is shown in Fig. 3. Here, Gibbs ensemble simulations are performed for a mixture with a site-site interaction energy of $\varepsilon_{a, b}^{*}=14.5$; the data is reported in Tables I and II. The region of closed-loop immiscibility can be clearly observed, bounded by both an UCST and a LCST. No lowtemperature regions of gas-liquid coexistence were isolated for this system. The SAFT-VR predictions at a pressure of $P^{*}=1.28$ with site-site interactions of $\varepsilon_{a, b}^{*}=14.5$ and $\varepsilon_{a, b}^{*}$ $=15$ are also shown. For $\varepsilon_{a, b}^{*}=14.5$ the theory predicts a region of liquid-liquid immiscibility bounded by an UCST and regions of low-temperature gas-liquid coexistence at temperatures below the three-phase line. Increasing the strength of the site-site interaction to $\varepsilon_{a, b}^{*}=15$ leads to the prediction of closed-loop immiscibility, with upper and lower boundaries which are similar to those obtained using Gibbs ensemble simulation. It is encouraging to observe the 
TABLE II. Fraction of molecules bonded in each of the two phases $X_{a}$ and $X_{b}$ obtained from NPT Gibbs ensemble Monte Carlo simulations for the symmetrical mixture of square wells with a single interaction site of reduced depth $\varepsilon_{a, b}^{*}=14.5$ at a reduced pressure of $P^{*}=1.28$. See Table I for details.

\begin{tabular}{cccc}
\hline \hline $\mathrm{N}$ & $T^{*}$ & $X_{a}$ & $X_{b}$ \\
\hline 1728 & 1.55 & $0.423 \pm 0.030$ & $0.416 \pm 0.033$ \\
1728 & 1.56 & $0.297 \pm 0.028$ & $0.305 \pm 0.031$ \\
1728 & 1.57 & $0.308 \pm 0.030$ & $0.321 \pm 0.038$ \\
1728 & 1.58 & $0.246 \pm 0.018$ & $0.257 \pm 0.027$ \\
1728 & 1.59 & $0.242 \pm 0.026$ & $0.239 \pm 0.030$ \\
1728 & 1.60 & $0.220 \pm 0.028$ & $0.214 \pm 0.031$ \\
1728 & 1.61 & $0.193 \pm 0.020$ & $0.187 \pm 0.022$ \\
1728 & 1.62 & $0.185 \pm 0.023$ & $0.190 \pm 0.027$ \\
1728 & 1.63 & $0.181 \pm 0.025$ & $0.181 \pm 0.031$ \\
1000 & 1.64 & $0.182 \pm 0.034$ & $0.215 \pm 0.029$ \\
1000 & 1.65 & $0.157 \pm 0.031$ & $0.211 \pm 0.032$ \\
1000 & 1.68 & $0.170 \pm 0.030$ & $0.194 \pm 0.027$ \\
1000 & 1.70 & $0.164 \pm 0.032$ & $0.173 \pm 0.032$ \\
1000 & 1.72 & $0.162 \pm 0.029$ & $0.147 \pm 0.036$ \\
1000 & 1.74 & $0.134 \pm 0.026$ & $0.155 \pm 0.023$ \\
1000 & 1.75 & $0.156 \pm 0.027$ & $0.159 \pm 0.025$ \\
1000 & 1.76 & $0.150 \pm 0.030$ & $0.161 \pm 0.026$ \\
1000 & 1.78 & $0.141 \pm 0.027$ & $0.170 \pm 0.024$ \\
1000 & 1.80 & $0.130 \pm 0.025$ & $0.139 \pm 0.027$ \\
1728 & 1.81 & $0.141 \pm 0.023$ & $0.146 \pm 0.022$ \\
1728 & 1.82 & $0.140 \pm 0.023$ & $0.144 \pm 0.022$ \\
1728 & 1.83 & $0.146 \pm 0.016$ & $0.136 \pm 0.015$ \\
1728 & 1.84 & $0.137 \pm 0.017$ & $0.134 \pm 0.017$ \\
1728 & 1.85 & $0.140 \pm 0.019$ & $0.143 \pm 0.017$ \\
1728 & 1.86 & $0.140 \pm 0.021$ & $0.147 \pm 0.018$ \\
\hline \hline
\end{tabular}

existence of a closed-loop of immiscibility for this system with a single association site, both by GEMC simulation and with the SAFT-VR equation of state. The use of the Wegner expansion of Eq. (47) which includes a first correction to scaling, with $\lambda_{\text {switch }}=0.1$ and $T_{\text {switch }}^{*}=1.63$, to fit the simulation data, is seen to give an excellent description of the shape of the closed loop. The estimated values for the critical temperatures, together with the values of the coefficients $B_{0}$ and $B_{1}$ obtained are given in Table III.

A temperature-composition slice obtained at a higher pressure of $P^{*}=1.48$ by Gibbs ensemble simulation is shown in Fig. 4. As for the case of the $P^{*}=1.28$ slice, the strength of the site-site interaction is fixed at $\varepsilon_{a, b}^{*}=14.5$, and the simulation results indicate the presence of a region of

TABLE III. Estimated critical constants for the symmetrical square-well mixture with a single bonding site of reduced energy $\varepsilon_{a, b}^{*}=14.5$ at a reduced pressure of $P^{*}=1.28$. The "error' denotes the maximum possible error which is estimated from the combined errors in the compositions obtained from the simulation data.

\begin{tabular}{lcc}
\hline \hline & UCST & LCST \\
\hline$T_{c}^{*}(N)$ & $1.915 \pm 0.689$ & $1.557 \pm 0.009$ \\
$B_{0}$ & $2.32 \pm 1.85$ & $3.00 \pm 0.21$ \\
$B_{1}$ & $-2.61 \pm 3.20$ & $-4.94 \pm 0.54$ \\
\hline \hline
\end{tabular}

TABLE IV. Estimated critical constants for the symmetrical square-well mixture with a single bonding site of reduced energy $\varepsilon_{a, b}^{*}=14.5$ at a reduced pressure of $P^{*}=1.48$. See Table III for details.

\begin{tabular}{lcc}
\hline \hline & UCST & LCST \\
\hline$T_{c}^{*}(N)$ & $2.068 \pm 1.038$ & $1.466 \pm 0.239$ \\
$B_{0}$ & $2.01 \pm 2.27$ & $2.03 \pm 0.89$ \\
$B_{1}$ & $-1.45 \pm 3.01$ & $-1.51 \pm 1.12$ \\
\hline \hline
\end{tabular}

closed-loop immiscibility. No low-temperature immiscible regions are found at this pressure using the GEMC method. For this higher pressure the extent of the closed-loop region has increased from that of the $P^{*}=1.28$ state, which is in line with the theoretical prediction of the effect of pressure on the closed loop (as described later). The prediction of phase behavior obtained with the SAFT-VR equation for two different interaction energies of $\varepsilon_{a, b}^{*}=14.5$ and $\varepsilon_{a, b}^{*}=15$ is also shown. In this case, the theoretical results do not indicate the existence of a LCST for the system at these conditions. The narrow ranges of pressure and interaction strength which result in a region of closed-loop immiscibility is thus illustrated: for a constant interaction strength of $\varepsilon_{a, b}^{*}=15$ an increase of pressure from $P^{*}=1.28$ (Fig. 3) to $P^{*}=1.48$ (Fig. 4) the theory predicts the loss of the LCST for the system since the region of liquid-liquid immiscibility merges with the low-temperature gas-liquid coexistence regions. The fit of the simulation data obtained using the Wegner expansion of Eq. (47) with $\lambda_{\text {switch }}=0.1$ and $T_{\text {switch }}^{*}=1.70$ is also shown in Fig. 4. As for the lower pressure case, this method accurately describes the form of coexistence curve. The corresponding estimates of the critical temperatures and parameters are given in Table IV.

In order to monitor the amount of association within the system, we calculate the fraction of molecules bonded $X_{j}$. Bonding only occurs when two unlike species are such that their off-center interaction sites overlap. The extent of association for the simulations performed at $P^{*}=1.28$ and $P^{*}$ $=1.48$ are shown in Fig. 5. For temperatures close to the UCST approximately $10 \%$ of the molecules are bonded and a dramatic increase of association is observed as the LCST is approached. This indicates that the low-temperature miscibiltiy of the system below the LCST is directly related to the association of unlike species.

The effect of varying the strength of the site-site interaction on the phase behavior of the model system as predicted by the SAFT-VR equation of state is shown in the PT projection of Fig. 6 (also see Ref. [5]). The vapor pressure curves of the pure components lie on the same line due to the symmetry of the mixture. The dashed curve which originates at the vapor-liquid critical point of the pure component and moves to higher pressures and temperatures represents the gas-liquid critical line. For a site-site interaction energy of $\varepsilon_{A B}^{*}=15$ this gas-liquid critical line goes through a minimum in temperature close to the critical point of the pure component. The critical line then moves to higher pressures, reaching a maximum before ending at the three-phase line. The continuous curve which originates at high pressures is the three-phase line for the mixture, along which two liquid 

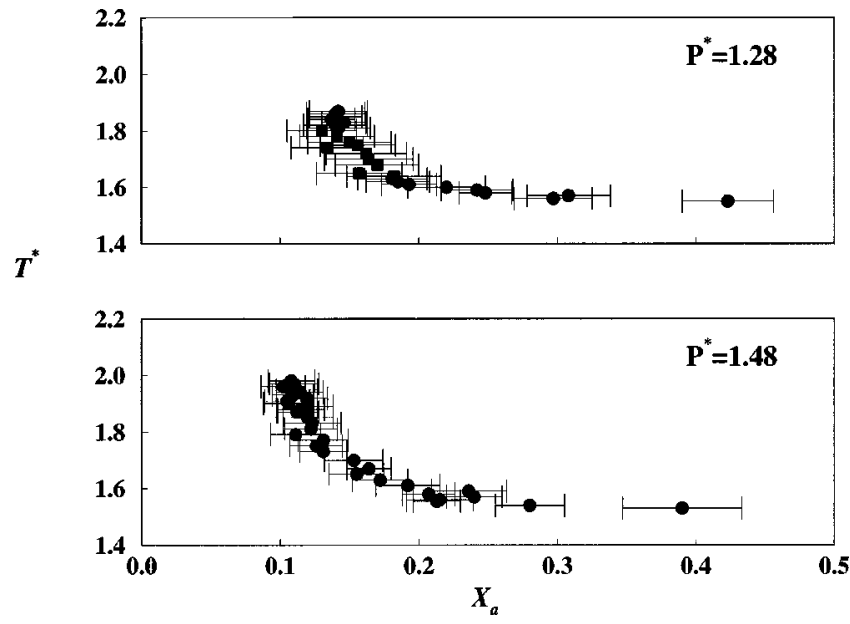

FIG. 5. Fraction of the total number of molecules bonded in phase $a, X_{a}$, as a function of reduced temperature $T^{*}=k T / \varepsilon$ for the symmetrical square-well mixture at a reduced pressure of $P^{*}$ $=P \sigma^{3} / \varepsilon=1.28$ and $P^{*}=P \sigma^{3} / \varepsilon=1.48$, with a bonding interaction of $\varepsilon_{a, b}^{*}=\varepsilon_{a, b} / \varepsilon=14.5$. The degree of association is shown for a single phase which, due to the symmetry of interactions, is the same for both coexisting phases. The squares correspond to the GEMC data for a system of $N=1000$ particles, and the circles to a system of $N=1728$ particles. The error bars correspond to one standard deviation.

phases and one "gas" phase coexist. The dashed curve which extends to higher temperatures going through a minimum in pressure is the liquid-liquid critical line. This line forms the boundary of the closed-loop region for this mixture for pressures between where it meets the three-phase line and

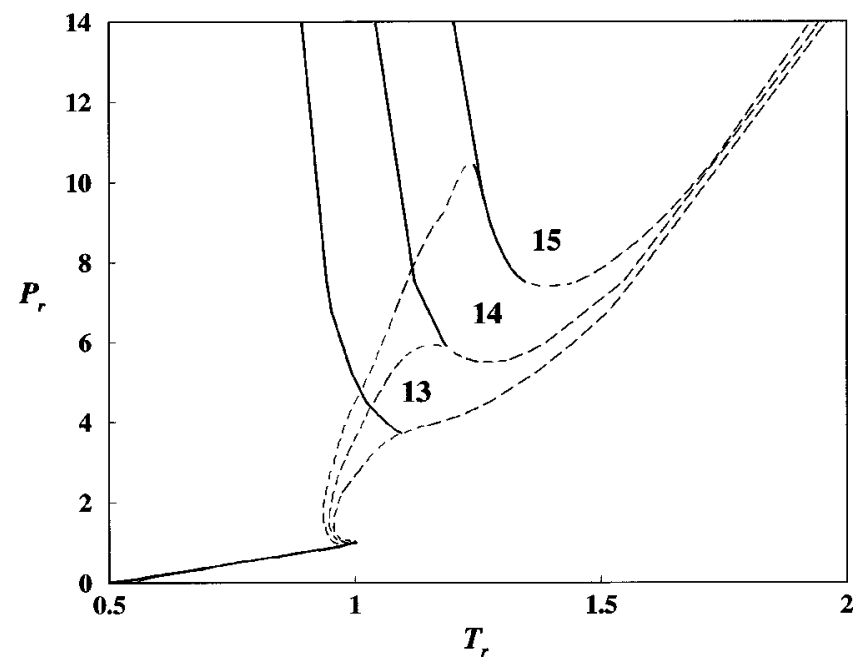

FIG. 6. Pressure-temperature projection for the binary associating square-well mixture with a range of bonding strengths of $\varepsilon_{a, b}^{*}$ $=13,14$, and 15 obtained using the SAFT-VR equation of state. The reduced pressure $P_{r}=P^{*} / P_{c}^{*}$ and reduced temperature $T_{r}$ $=T^{*} / T_{c}^{*}$ are used. The solid curve at low pressures and temperatures corresponds to the vapor-liquid curve of the pure component, while the solid curve at high pressures and temperatures corresponds to the three-phase line of the mixture. The dashed curves correspond to the critical lines, gas-liquid at low pressures and temperatures, and liquid-liquid at high pressures and temperatures. The curves are labeled with the corresponding value of the site-site interaction energy.

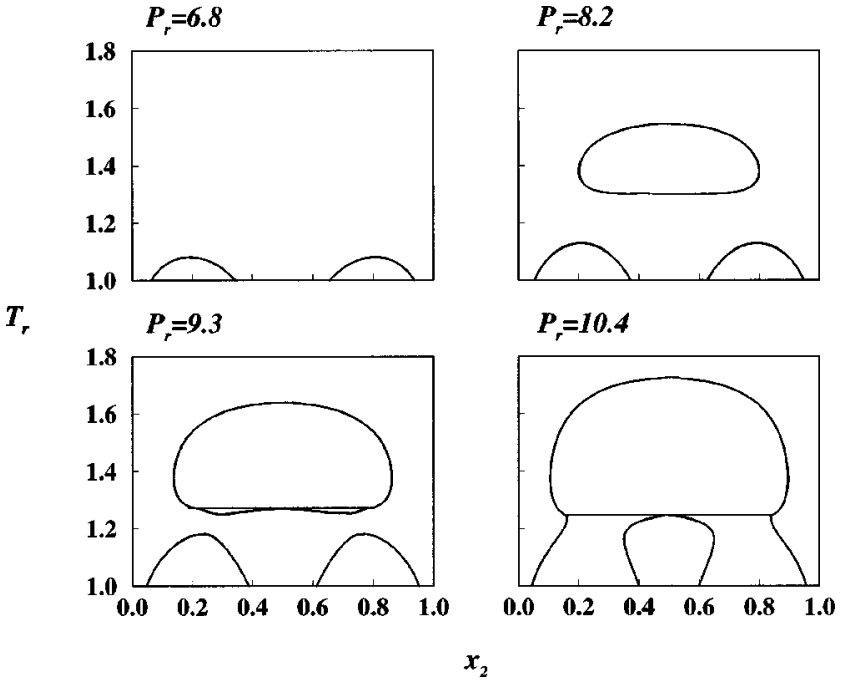

FIG. 7. Temperature-composition slices of the coexistence region for the symmetrical square-well mixture with $\varepsilon_{a, b}^{*}=15$ at a series of reduced pressures $P_{r}=6.8,8.2,9.3$, and 10.4 using the SAFT-VR equation of state. The reduced pressure $P_{r}=P^{*} / P_{c}^{*}$ and reduced temperature $T_{r}=T^{*} / T_{c}^{*}$ are used. The curves are labeled with the corresponding value of the reduced pressure and the horizontal lines correspond to the three-phase lines.

the minimum in the liquid-liquid critical line. In this region the system is seen to possess two liquid-liquid critical points, the LCST at lower temperatures and the UCST at higher temperatures. Decreasing the association strength from $\varepsilon_{a, b}^{*}$ $=15$ to $\varepsilon_{a, b}^{*}=14$ shrinks the minimum in the liquid-liquid critical line, and hence the extent of closed-loop immiscibility exhibited by the system. A further decrease of the magnitude of the interaction energy to $\varepsilon_{a, b}^{*}=13$ leads to the disappearance of the minimum in the liquid-liquid critical line; the three-phase line meets the gas-liquid and the liquid-liquid critical lines before either go through a maximum (or minimum) in pressure. In this case the system does not exhibit closed-loop immiscibility. When the strength of the bonding interaction is zero the only feature of the phase diagram is the region of liquid-liquid immiscibility below an UCST, as illustrated with the simpler SAFT-HS approach [5] and GEMC simulation [48,44]. Conversely, increasing the strength of the site-site interaction beyond $\varepsilon_{a, b}^{*}=15$, leads to complete liquid-liquid miscibiliy, so that the system only exhibits gas-liquid critical lines. Use of the SAFT-VR methodology to evaluate the mean-attractive energy and its derivatives (as opposed to the SAFT-HS treatment of the attractive interactions at the van der Waals level [5]) decreases the effect of the reduced site-site interaction $\varepsilon_{a, b}^{*}$, thus reducing the extent of the closed-loop immiscibility which is observed for specific values of the bonding energy.

The effect of a variation in pressure on the extent of the closed-loop region of the binary square-well mixture is illustrated in Fig. 7 for a series of constant pressure slices of a system with $\varepsilon_{a, b}^{*}=15$. For a reduced pressure of $P_{r}=6.8$ the system is seen to be miscible at all temperatures above $T_{r}=1.1$, indicating that this pressure is well below the minimum in pressure of the liquid-liquid critical line. The closedloop region appears when the pressure of the system is increased to $P_{r}=8.2$, and a further increase in pressure to 
$P_{r}=9.3$ gives rise to the the gas-liquid-liquid three-phase line. At a higher pressure of $P_{r}=10.4$ this gas-liquid region merges with the low temperature gas-liquid region and the system only possesses a single critical point at these pressures.

\section{CONCLUSIONS}

The associating model system examined in this work can be considered as a prototype for a real fluid which exhibits the phenomenon of closed-loop immiscibility as a result of hydrogen bonding interactions. The simplistic nature of the model allows for a clear understanding of this type of phase behavior. The phase behavior of the associating system is shown to contain regions of closed-loop liquid-liquid immiscibility for specific values of the site-site interaction energy both with GEMC simulations and the SAFT-VR equation of state. Association between the unlike species in the mixture results in the low-temperature miscibiliy of this model system. For weak interaction strengths no LCST exists. The SAFT-VR approach predicts that the LCST and the UCST merge at a specific temperature and pressure upon increasing the strength of the bonding interaction; above this the system has no regions of liquid-liquid immiscibility.

Both the computer simulation and the SAFT-VR approach illustrate that the extent of reentrance in such a model depends on the pressure and temperature of the system and also on the strength of the site-site interaction energy. The agreement between the two approaches indicates the validity of the use of the SAFT-VR equation of state in the prediction of the phase behavior of such associating systems.

Despite the direct correlation observed in this work between the directional association of unlike components and the existence of low-temperature miscibility below a LCST several studies have illustrated the existence of closed-loop regions for systems such as Lennard-Jones molecules [52] and attractive spheres [53] where the interactions are spherically symmetrical. The approximate theories used in these studies predict unrealistic LCSTs at very low temperatures for densities which are characteristic of the solid phase. Although it is possible that isotropic interactions could give rise to reentrance under such unphysical conditions, anisotropic interactions such as hydrogen bonding are the central feature of systems which exhibit closed-loop behavior, a fact which is borne out by experiment. Canongia Lopes [54] has recently found type VI phase behavior in mixtures of LennardJones molecules with the GEMC technique, but in his case the driving force for the reentrant liquid-liquid immiscibility is a cross interaction diameter that is smaller than the arithmetic mean of the like diameters; there is thus an increase in translational entropy (better packing) when the system mixes at lower temperatures. In this context it is also important to mention related simulation studies of closed-loop immiscibilty for lattice models with directional interactions in both bulk [55] and confined [56] systems; as for the analytical studies with lattice models, closed-loop behavior is observed for suitable choices of the intermolecular parameters. Reentrance can also be seen in charged-stabilized colloidal suspensions [57], which again is not caused by hydrogen bonding or molecular association; the complex interplay between Coulombic and entropic effects gives rise to the phase behavior. The relation between directional attractive interactions and closed-loop immiscibility of two fluid phases is clearly defined experimentally in hydrogen-bonded liquids such as water and alcohol, and it is this feature of associating fluids which we focus on in this work.

Reentrant phase behavior is of course not restricted to simple fluids. Reentrant liquid crystalline nematic phases can be seen as a consequence of the delicate temperature dependence of hydrogen bonding (e.g., see Refs. [58]). In fact molecular association and dipole pairing is often used to explain reentrant behavior [59]. Hydrogen bonding offers a number of new possibilities to control the phase transitions in liquid crystalline and polymeric systems.

\section{ACKNOWLEDGMENTS}

L.A.D. thanks the Engineering and Physical Sciences Research Council (EPSRC) and BP for financial support. We also acknowledge support from the Royal Society, the EPSRC, the European Union, and the DGICYT (PB94-1442) for computer hardware.
[1] W. Dolgolenko, Z. Phys. Chem. (Leipzig) 62, 499 (1908).

[2] R. J. L. Andon and J. D. Cox, J. Chem. Soc. 1952, 4601 (1952)

[3] J. C. Lang and R. D. Morgan, J. Chem. Phys. 73, 5849 (1980).

[4] J. S. Walker and C. A. Vause, Sci. Am. 256, 90 (1987).

[5] G. Jackson, Mol. Phys. 72, 1365 (1991).

[6] M. S. Wertheim, J. Stat. Phys. 35, 19 (1984).

[7] M. S. Wertheim, J. Stat. Phys. 35, 35 (1984).

[8] M. S. Wertheim, J. Stat. Phys. 42, 459 (1986).

[9] M. S. Wertheim, J. Stat. Phys. 42, 477 (1986).

[10] G. Jackson, W. G. Chapman, and K. E. Gubbins, Mol. Phys. 65, 1 (1988).

[11] W. G. Chapman, G. Jackson, and K. E. Gubbins, Mol. Phys. 65, 1057 (1988).

[12] W. G. Chapman, K. E. Gubbins, G. Jackson, and M. Radosz, Fluid Phase Equilibria 52, 31 (1989).

[13] W. G. Chapman, K. E. Gubbins, G. Jackson, and M. Radosz,
Ind. Eng. Chem. Res. 29, 1709 (1990).

[14] L. A. Davies, G. Jackson, and L. F. Rull, Phys. Rev. Lett. 82, 5285 (1999).

[15] A. Gil-Villegas, A. Galindo, P. J. Whitehead, S. J. Mills, G. Jackson, and A. N. Burgess, J. Chem. Phys. 106, 4168 (1997).

[16] A. Galindo, L. A. Davies, A. Gil-Villegas, and G. Jackson, Mol. Phys. 93, 241 (1998).

[17] R. L. Scott and P. H. van Konynenburg, Discuss. Faraday Soc. 49, 87 (1970).

[18] P. H. van Konynenburg and R. L. Scott, Philos. Trans. R. Soc. London, Ser. A 298, 495 (1980).

[19] D. G. Green and G. Jackson, J. Chem. Phys. 97, 8672 (1992); I. Nezbeda, J. Pavlíček, J. Kolafa, A. Galindo, and G. Jackson, Fluid Phase Equilibria 160, 193 (1999).

[20] M. N. García-Lisbona, A. Galindo, G. Jackson, and A. N. Burgess, Mol. Phys. 93, 57 (1998).

[21] M. N. García-Lisbona, A. Galindo, G. Jackson, and A. N. Bur- 
gess, J. Am. Chem. Soc. 120, 4191 (1998).

[22] J. A. Barker, and D. Henderson, J. Chem. Phys. 47, 2856 (1967).

[23] J. A. Barker and D. Henderson, J. Chem. Phys. 47, 4714 (1967).

[24] J. A. Barker and D. Henderson, Rev. Mod. Phys. 48, 587 (1976).

[25] A. Z. Panagiotopoulos, Mol. Phys. 61, 813 (1987).

[26] A. Z. Panagiotopoulos, N. Quirke, M. R. Stapleton, and D. J. Tildesley, Mol. Phys. 63, 527 (1988).

[27] K. E. Gubbins, Mol. Simul. 2, 223 (1989).

[28] A. Z. Panagiotopoulos, Mol. Simul. 9, 1 (1992).

[29] L. F. Rull, G. Jackson, and B. Smit, Mol. Phys. 85, 435 (1995).

[30] M. P. Allen and D. J. Tildesley, Computer Simulation of Liquids (Clarendon, Oxford, 1987).

[31] B. Widom, J. Chem. Phys. 39, 2802 (1963).

[32] B. Smit and D. Frenkel, Mol. Phys. 68, 951 (1989).

[33] L. L. Lee, Molecular Thermodynamics of Nonideal Fluids (Butterworth, Washington, DC, 1988).

[34] T. Boublík, J. Chem. Phys. 53, 471 (1970).

[35] G. A. Mansoori, N. F. Carnahan, K. E. Starling, and T. W. Leland, J. Chem. Phys. 54, 1523 (1971).

[36] N. F. Carnahan and K. E. Starling, J. Chem. Phys. 51, 635 (1969).

[37] T. M. Reed, and K. E. Gubbins, Applied Statistical Mechanics (McGraw-Hill, New York, 1973).

[38] W. H. Press, B. P. Flannery, S. A. Teukolsky, and W. T. Vetterling, Numerical Recipes, 2nd ed. (Cambridge University Press, Cambridge, England 1992).

[39] B. Smit, Ph. de Smedt, and D. Frenkel, Mol. Phys. 68, 931 (1989).

[40] F. J. Wegner, Phys. Rev. B 5, 4529 (1972).

[41] J. V. Sengers and J. M. H. Levelt Sengers, Annu. Rev. Phys. Chem. 37, 189 (1986).
[42] R. R. Singh and K. S. Pitzer, J. Chem. Phys. 90, 5742 (1989).

[43] L. Vega, E. de Miguel, L. F. Rull, G. Jackson, and I. A. McLure, J. Chem. Phys. 96, 2296 (1992).

[44] D. G. Green, G. Jackson, E. de Miguel, and L. F. Rull, J. Chem. Phys. 101, 3190 (1994).

[45] L. P. Kadanoff, Phase Transitions and Critical Phenomena, edited by C. Domb and M. S. Green (Academic, New York, 1976), Vol. 5A, pp. 1-34.

[46] H. E. Stanley, Introduction to Phase Transitions and Critical Phenomena (Oxford University Press, Oxford, 1971).

[47] K. K. Mon and K. Binder, J. Chem. Phys. 96, 6989 (1992).

[48] J. R. Recht and A. Z. Panagiotopoulos, Mol. Phys. 80, 843 (1993).

[49] E. de Miguel, Phys. Rev. E 55, 1347 (1997).

[50] A. Z. Panagiotopoulos, Int. J. Thermophys. 15, 1057 (1994).

[51] E. de Miguel, E. M. del Rio, and M. M. Telo da Gamma, J. Chem. Phys. 103, 6188 (1995).

[52] V. A. Mazur, L. Z. Boshkov, and V. B. Fedorov, Dokl. Akad. Nauk SSSR 282, 137 (1985); L. Z. Boshkov and V. A. Mazur, Russ. J. Phys. Chem. 60, 16 (1986); L. Z. Boshkov, Dokl. Akad. Nauk SSSR 294, 901 (1987).

[53] L. V. Yelash and T. Kraska, Ber. Bunsenges. Phys. Chem. 102, 213 (1998)

[54] J. N. Canongia Lopes, Mol. Phys. 96, 1649 (1999).

[55] M. Kotelyanskii, B. Veytsman, and S. K. Kumar, Phys. Rev. E 58, R12 (1998).

[56] M. Kotelyanskii and S. K. Kumar, Phys. Rev. Lett. 80, 1252 (1998).

[57] R. van Roij, M. Dijkstra, and J.-P. Hansen, Phys. Rev. E 59, 2010 (1999).

[58] R. P. Sear and G. Jackson, Mol. Phys. 83, 961 (1994): Phys. Rev. Lett. 74, 4261 (1995).

[59] P. E. Cladis, Mol. Cryst. Liq. Cryst. 85, 165 (1988). 